\title{
EVALUACIÓN DE LINEAS DE REFERENCIA DE POTENCIAL DE TALLO Y MÁXIMA CONTRACCIÓN DIARIA DE RAMA PARA LA PROGRAMACIÓN DEL RIEGO EN CEREZO
}

\author{
Blanco Montoya, V. (1), Blaya Ros, P.J. (1), Temnani, A. (1), Torres Sánchez, R. (2), González Teruel, J.D. (2), \\ Soto Valles, F (2), Toledo Moreo, A.B. (2), Jiménez Buendía, M. (2), Domingo Miguel, R. (1)
}

1 Dpto. de Ingeniería Agronómica, Universidad Politécnica de Cartagena. victor.blanco@upct.es; pedro.blaya@upct.es; abdelmalek91@gmail.com; rafael.domingo@upct.es ${ }^{2}$ Dpto. de Automática, Ingeniería Eléctrica y Tecnología Electrónica, Universidad Politécnica de Cartagena. roque.torres@upct.es; juando.gonzalez@upct.es; pencho.soto@upct.es; ana.toledo@upct.es; manuel.jimenez@upct.es.

\section{Introducción}

Conocer el estado hídrico de la planta es clave para reducir los volúmenes de agua a aplicar en relación a sus necesidades máximas sin penalizar la producción. En árboles frutales, el potencial hídrico de tallo a mediodía se considera el indicador de referencia; sin embargo, presenta la limitación de que no se puede automatizar. Por ello, indicadores que pueden ser obtenidos a partir de medidas continuas en tiempo real y de fácil automatización, como la máxima contracción diaria de rama, tienen gran interés.

\section{OBJETIVO: Elaborar y analizar las líneas de referencia $\Psi_{t}$ y MCD en relación a variables meteorológicas.}

\section{Materiales y Métodos}

\section{Condiciones Experimentales}

Cerezo [Prunus avium (L.)] “Prime Giant”/SL64

Localización: Finca Toli, Jumilla, Región de Murcia

Plantación (2000): 667 árboles ha-1

Riego por goteo: 3 emisores $\left(4 \mathrm{~L} \mathrm{~h}^{-1}\right)$ por árbol

Calidad agua de riego: $\mathrm{EC}=0.8 \mathrm{dS} / \mathrm{m}$

Campañas: 2015 y 2016

Diámetro rama medio: $9,03 \pm 0,68 \mathrm{~cm}$

Condiciones meteorológicas: Clima Mediterráneo.

\begin{tabular}{|c|c|c|} 
& $E_{0}$ acumulada $(\mathrm{mm})$ & Agua aplicada $(\mathrm{mm})$ \\
\hline 2015 & 1272 & 692 \\
\hline 2016 & 1220 & 703 \\
\hline
\end{tabular}

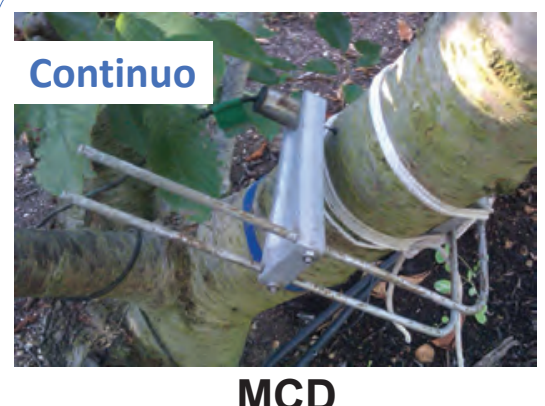

Máxima Contracción Diaria de Rama

Los árboles fueron regados durante toda la campaña (Abril - Noviembre) para mantener condiciones no limitantes de agua en suelo al $110 \%$ ETC.

\section{Resultados}

$\Psi_{\mathrm{t}}$ y MCD resultaron muy dependientes de las variables climáticas, particularmente del déficit de presión de vapor (DPV).

Relaciones potencial de tallo a mediodía $\left(\Psi_{t}\right)$ y máxima contracción diaria $(\mathrm{MCD})$ versus variables meteorológicas. Relaciones expresadas como: $y=a x+b$.

\begin{tabular}{|c|c|c|c|c|c|c|c|c|c|c|c|}
\hline$\Psi$ & $\mathbf{a}$ & b & $r^{2}$ & $\mathbf{n}$ & ecm & MCD & a & b & $r^{2}$ & $\mathbf{n}$ & ecm \\
\hline$\Psi_{t}$ Vs. $T_{m}$ & & & & & & MCD vs. $T_{m}$ & & & & & \\
\hline 2015 & $-0,026$ & $-0,08$ & 0,73 & 16 & 0,0059 & 2015 & 14,9 & $-134,1$ & 0,59 & 192 & 3556 \\
\hline 2016 & $-0,026$ & $-0,10$ & 0,66 & 14 & 0,0078 & 2016 & 10,9 & $-52,5$ & 0,46 & 182 & 2694 \\
\hline$\Psi_{\mathrm{t}}$ vs. $T_{\operatorname{Max}}$ & & & & & & MCD vs. $T_{\operatorname{Max}}$ & & & & & \\
\hline 2015 & $-0,021$ & $-0,019$ & 0,75 & 16 & 0,0055 & 2015 & 13,0 & $-192,5$ & 0,69 & 192 & 2664 \\
\hline 2016 & $-0,022$ & $-0,014$ & 0,69 & 14 & 0,0069 & 2016 & 9,3 & $-92,1$ & 0,57 & 182 & 2145 \\
\hline$\Psi_{\mathrm{t}} \mathrm{v}_{\mathrm{.}}, \mathrm{ET}_{0}$ & & & & & & MCD vs. $\mathrm{ET}_{0}$ & & & & & \\
\hline 2015 & $-0,081$ & $-0,233$ & 0,64 & 16 & 0,2057 & 2015 & 42,1 & $-18,6$ & 0,65 & 192 & 3068 \\
\hline 2016 & $-0,067$ & $-0,287$ & 0,45 & 14 & 0,0124 & 2016 & 33,2 & 12,3 & 0,63 & 182 & 1800 \\
\hline$\Psi_{\mathrm{t}}$ VS. DPV & & & & & & MCD vs. DPV & & & & & \\
\hline 2015 & $-0,194$ & $-0,304$ & 0,84 & 16 & 0,0035 & 2015 & 105,6 & 7,2 & 0,75 & 192 & 2166 \\
\hline 2016 & $-0,180$ & $-0,326$ & 0,70 & 14 & 0,0068 & 2016 & 75,6 & 48,7 & 0,65 & 182 & 1745 \\
\hline
\end{tabular}

DPV fue la variable con menor ecm.

DPV fue capaz de responder

entre el 70 y el $84 \%$ de la variabilidad de $\Psi \mathrm{t}$

entre el 65 y el $75 \%$ de la variabilidad de MCD
Orden de dependencia de $\boldsymbol{\Psi}_{\mathbf{t}}$ y $\mathbf{M C D}$ de las v. meteorológicas:

$\Psi_{t}: \quad D P V>T_{\max }>T_{m}>E T_{0}$ $M C D: D P V>T_{\max }>E T_{0}>T_{m}$

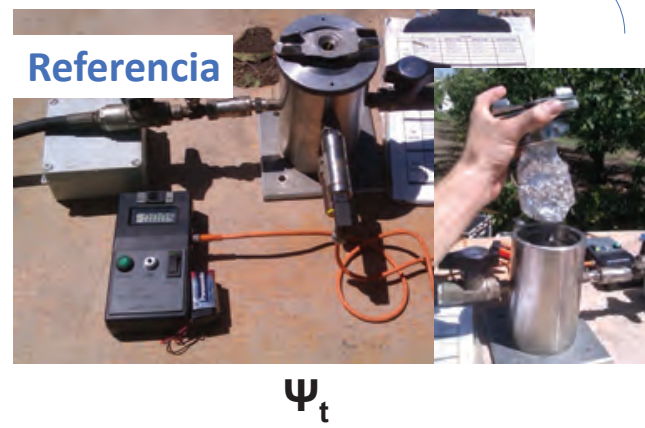

Potencial Hídrico de Tallo a Mediodía
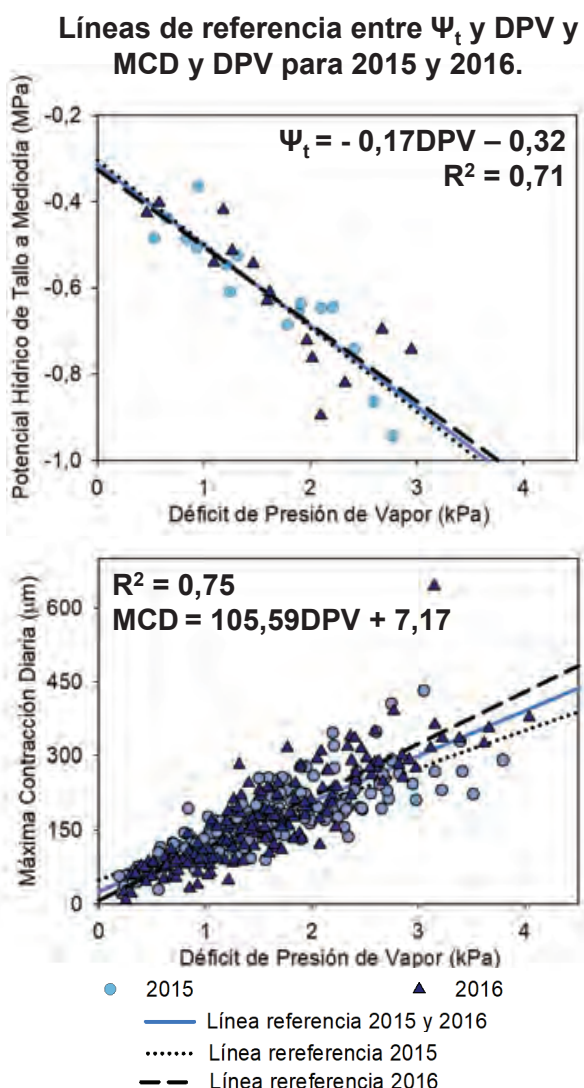

Agradecimientos

Este trabajo fue financiado por el Ministerio de Economía y Competitividad del Gobierno de España y fondos FEADER (ref: AGL2013-49047-C2-1-R y AGL201677282-C3-3-R) y por la "Fundación Séneca" de la Región de Murcia bajo el programa de Grupos de Excelencia 19895/GERM/15. Los autores agradecen a Pedro y Agustín Carrión-Guardiola, propietarios de "Finca Toli" todas las facilidades puestas a nuestro alcance para el correcto desarrollo de los ensayos. 